\title{
Development of Remote Temperature Monitoring and Fault Tolerant Reporting System for Acid Gases Removal Unit
}

\author{
${ }^{1}$ Omagbemi O.W., ${ }^{2}$ Nwokoye A.O.C. \\ ${ }^{1}$ Department of Physics, Federal University of Petroleum Resources, Effurum, Nigeria \\ ${ }^{2}$ Department of Physics and Industrial Physics, Nnamdi Azikiwe University, Awka, Nigeria \\ Email IDs: ${ }^{1}$ omagbemi.oghogho@ fupre.edu.ng, ${ }^{2}$ aoc.nwokoye@ unizik.edu.ng
}

\begin{abstract}
Industrial applications have evolved over the years with an ever-increasing need for optimal performance and as little down time as possible. To maintain integrity, stability, safety and fault finding with tolerant schemes were introduced to many industrial productions and instrumentation techniques. This work designs a fault diagnostic and tolerant (FDT) scheme; faults were diagnosed based on deviation from the normal working mode of primary components and automatically disengaged the system from supply voltage with alerting information for immediately action. The FDT was incorporated into an existing Proportional-IntegralDerivative (PID) control scheme. The FDT was also equipped with a remote monitoring system using Short Messaging Service (SMS) using a Sim800 to alert a remote system custodian on the occurrence of a fault and appropriate unit with components that is faulty. The fault diagnosis and tolerant control system with remote monitoring was then applied to a prototype acid gas removal system with a set point of $40^{\circ} \mathrm{C}$ with $38^{\circ} \mathrm{C}$ to $42^{\circ} \mathrm{C}$. The FDT was able to maintain the control objective when faults occurred and the SMS messaging initiated when faults were simulated on Proteus.
\end{abstract}

Keywords: Fault Diagnosis, Primary components, remote monitoring and robust system.

\section{INTRODUCTION}

In industrial applications, Fault Tolerant Control (FTC) systems are critical in order to preserve the systems integrity, maintain safety and stability. FTC are control systems that are proficient in maintaining the overall stability of a control system by tolerating component failures within the system. FTCs are designed to adapt to each systems peculiarity by postulating failures that can occur at each system component. These postulated failures are meant to be accommodated by the FTC as the stability of the system is maintained. [1]

FTC systems can either be active or passive. Passive FTC utilizes the postulated failures to create a robust system that maintains the systems integrity in the eventuality of a fault. A fault in this context refers to a deviation from the normal working principle of the individual system components [2] (Fekih and Pilla, 2009). In contrast to passive FTC, active FTC reacts to a detected fault by adopting a new or precomputed control law. Active FTCs takes passive FTC one step further from just using a robust system but also creates a solution (in real time) to enable the control system work at its optimal $[1,2]$.

FTCs are preceded by a fault detection and isolation (FDI) system which can be achieved using system component modelling to identify a possible fault. This process of identifying and isolating faults is crucial to the FTC systems as results obtained propels the next line of action for the FTC. The fault detection unit works based on preliminary analysis to classify the faults $[3,4]$ created a fault detection system using analytical redundancy relations and algorithms to determine faulty components in the isolation phase. [5] Wang, et al., (2008) designed a fault detection and isolation observer for sensor faults which was applied to a non-linear control system.

Remote monitoring systems have seen a rise in application with the increase of smart systems. Instrumentation and control have not been left behind in the adoption of remote communication techniques. From satellite relay systems in the 70 s to a more real time approach to offsite monitoring of industrial processes, remote reporting has proved to have evolved over the years as the need for optimal performance in industries, health-care delivery, security and even education has risen [6]. Short Message Service (SMS) is widely available in the entire digital cellular network covering from $2 \mathrm{G}$ to the newly introduced $5 \mathrm{G}$ network which is reported to allow more than 160 characters and include several multimedia features.

Fault tolerant control (FTC) systems aim to maintain acceptable performance in the presence of component/instrument faults. They are therefore designed to augment for faults that have already been detected and diagnosed and also provide a means of quickly isolating the faulty components to avoid damage of the component beyond repair or collapse of the entire control system $[7,8,9]$. 
SMS can reach areas covered by only $2 \mathrm{G}$ networks and does not require internet connection. Also, SMS is cheap and uses minimal network resources when sending messages [6]. This work designed a FTC system using postulated system component failures, incorporates this FTC into an already existing Proportional-Integral and Derivative (PID) control, and provides real time fault diagnosis and remote reporting via SMS.

\section{METHODOLOGY}

\subsection{Basic Block Diagram of Remote Temperature Monitoring Fault Tolerant Reporting System}

The Figure 1 is basic block diagram of remote temperature monitoring fault tolerant reporting system for acid gases removal unit consists of the following temperature sensors (LM35), SIM808 GSM module, graphic display unit, solid state actuator and control, acid gases removal chamber as load, buzzer, AC source and process controller (implement with Arduino $\mu$ controller).

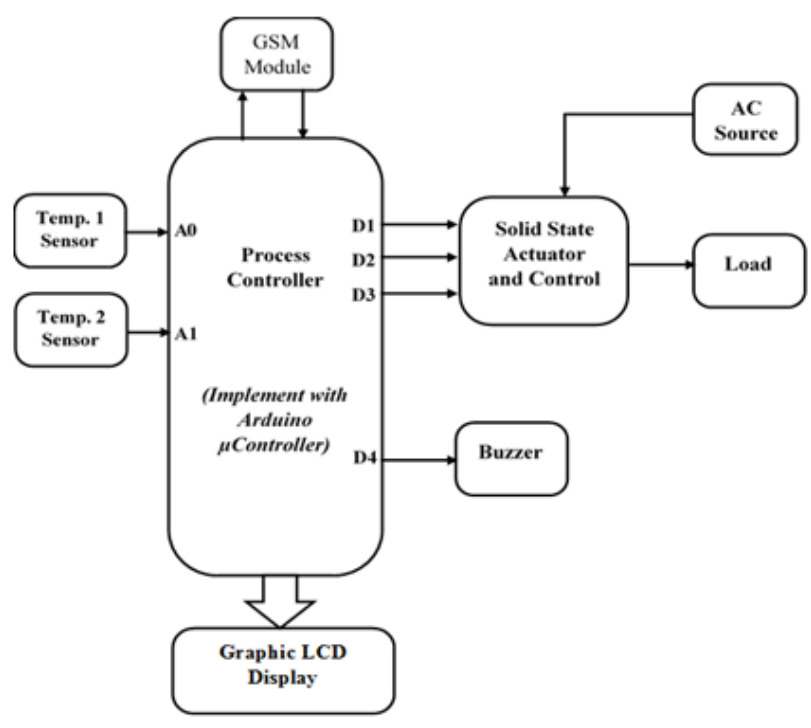

Figure 1: Block Diagram of Remote Temperature Monitoring Fault Tolerant Reporting System (RTMFTRS)

\subsection{Temperature Sensor Unit}

The LM35 is a semiconductor temperature. It has very good linearity and sensitivity is $10 \mathrm{mV} /{ }^{\circ} \mathrm{C}$ for a simple connection it required little or no external component. If properly biased the temperature range from $-15{ }^{\circ} \mathrm{C}$ to 150 ${ }^{\circ} \mathrm{C}$, but for simple connection it ranges from $2{ }^{\circ} \mathrm{C}$ to $150{ }^{\circ} \mathrm{C}$ $[10,11]$.

\subsection{Solid State Actuator and Control Unit}

The solid-state actuator and control section consists of DIAC optocoupler, Infrared optocoupler, and TRIAC.

\subsubsection{Infrared Optocoupler}

An IR sensor consists of an IR LED and an IR Photodiode; together they are called Photo-Coupler or Opto-Coupler (Figure 2). The Infrared isolation sensor has a built-in IR transmitter and IR receiver. An infrared Transmitter is a lightemitting diode (LED) that emits infrared radiation. Hence, they are called IR LEDs. Infrared receivers are also called infrared sensors as they detect the radiation from an IR transmitter. IR receivers come in the form of photodiodes and phototransistors. Infrared Photodiodes are different from normal photodiodes as they detect only infrared radiation. When the voltage across is above $3.3 \mathrm{~V}$ either AC or DC the infrared LED emit radiation and falls on the base IR phototransistor receiver that is power by $5 \mathrm{~V}$ DC conductor, current flow from collector to IR phototransistor. A $47 \mathrm{k} \Omega$ resistor was connected from ground to emitter of IR phototransistor, when its conductor there is a potential drop across $10 \mathrm{k} \Omega$ resistor and infrared LED is OFF the potential at emitter of IR phototransistor zero, $0 \mathrm{Vdc}$ is across the $47 \mathrm{k} \Omega$ resistor. This point is connected the digital output/input pin of Arduino for appropriate action. [12]

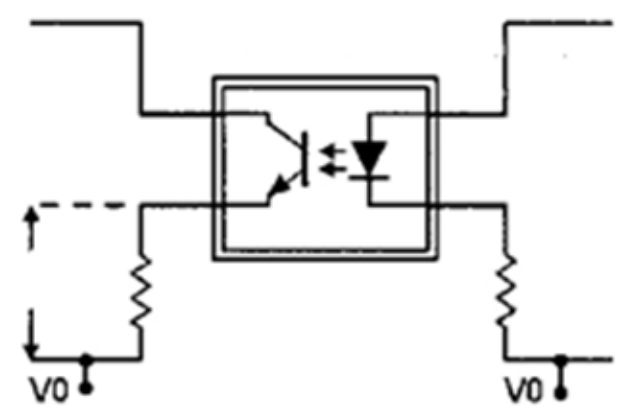

Figure 2: Optocoupler

\subsubsection{Triac and triac optocoupler}

A triac is a thyristor (SCR) that is capable of conducting in both halves of the mains AC cycle with zero-crossing detection allowing the load to receive full power without the heavy inrush currents when switching inductive loads (Figure $3)$.

Optocouplers and Opto-isolators (Figure 3) are great electronic devices that allow devices such as power transistors and triacs to be controlled from a PC's output port, digital switch or from a low voltage data signal such as that from a logic gate. The main advantage of opto-couplers is their high electrical isolation between the input and output terminals allowing relatively small digital signals to control much large $\mathrm{AC}$ voltages, currents and power.

An optocoupler can be used with both DC and AC signals with optocouplers utilizing a SCR (thyristor) or triac as the photo-detecting device are primarily designed for AC power- 
control applications. The main advantage of photo-SCRs and photo-triacs is the complete isolation from any noise or voltage spikes present on the AC power supply line as well as zero-crossing detection of the sinusoidal waveform which reduces switching and inrush currents protecting any power semiconductors used from thermal stress and shock.

In the circuit diagram in Figure 3, the pin 1 and 2 of the optocoupler are connected to a DC source through a switch and a resistor. The motor is connected to the AC supply through a TRIAC. The Photo-DIAC optocoupler (Figure 3) is used to trigger the TRIAC. When digital input/output pin (D14) is HIGH (turn ON), the IR LED inside the DIAC optocoupler will turn on and the light falls upon the DIAC. When the DIAC starts conducting, the TRIAC will be triggered, therefore the motor will turn on. [13]

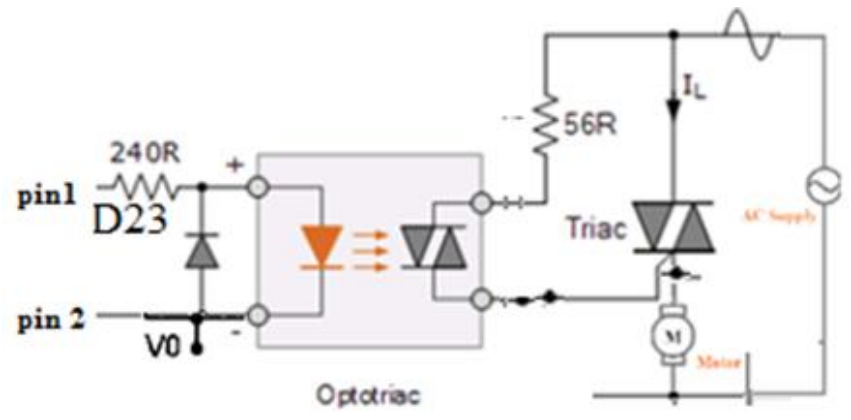

Figure 3: Optodiac and Triac Circuit

\section{COMMUNICATION UNIT}

SIM800 is a quad-band GSM/GPRS module that works on frequencies $850 \mathrm{MHz}$ GSM, 900MHz EGSM, 1800MHz DCS, and $1900 \mathrm{MHz}$ PCS. It also features GPRS multi-slot class 12/class 10 (optional), and supports CS-1, CS-2, CS-3, and CS-4 GPRS coding schemes.

It has one UART port. It also has one USB port that can be used for updating firmware and for debugging. Audio channels are also there, which include a microphone input and a receiver output. SIM800 has one SIM card interface. It integrates TCP/IP protocol.

SIM800 can be controlled/configured using simple AT commands. A host microcontroller can send AT commands over the UART interface and control the SIM800. SIM800 operates on a supply in the range of 3.4 to $4.4 \mathrm{~V}$. It can be used for sending/receiving messages, making calls, sending/receiving data over the internet. This makes it useful for applications such as home automation, agriculture automation and others. To communicate to the SIM800 a software serial library was to use any to the digital pin on Arduino Mega 2560 [14]. The sample of the code used in sending and receiving information is given below: \#include <Sim8001.h>

\#include <SoftwareSerial.h $>$ //is necesary for the library!!

Sim8001 Sim8001; //to declare the library

char* text;

char* number;

bool error; //to catch the response of sendSms

Sim800l.begin(); // initializate the library. text="Optotodiac fail"; //text for the message. number="08034277453"; //change to a valid number. error=Sim8001.sendSms(number,text); // OR //Sim8001.sendSms("08034277453"," Optotodiac fail ")

\section{PID TEMPERATURE CONTROLLER ALGORITHM}

The monitoring of temperature of acid removal unit was implement using proportional integral and derivative (PID). There are three primary components of a PID control loop. Each component is prefixed with a gain constant, and when added together, gives the instantaneous control value that used to drive unit. Typically, at input a voltage is generate to control unit, that contributing a particular voltage to the final output $[15,16]$.

A PID controller is a continuously calculating an error value as the difference between the desired setpoint (SP) and a measured process variable (PV) and applies a correction based on proportional, integral, and derivative terms (denoted $P, I$, and $D$ respectively) with summation output $u$ is given in equation below.

$$
u=\underbrace{K_{p} e}_{\begin{array}{c}
\text { Proportional } \\
\text { Term }
\end{array}}+\underbrace{K_{i} \int_{0}^{t} e d t}_{\begin{array}{c}
\text { Integral } \\
\text { Term }
\end{array}}+\underbrace{K_{d} \frac{d}{d t} e}_{\begin{array}{c}
\text { Differential } \\
\text { Term }
\end{array}}
$$

The controller in the continuous or analog domain makes it easier for us to realize what is going on. The conversion of equation 1 to discrete time or digital domain as shown in equation below:

$$
u[n]=K_{p} \times e[n]+K_{i} \times \sum_{k=0}^{n} e[k] T+K_{d} \times \frac{(e[n]-e[n-1])}{T}
$$

The equation 2 was implemeted using decrete time domain in Arduino code in expression shown belon below.

$/ *$ Intiallised the time for computation and the variable most be declared $* /$

unsigned long now $=$ millis ()$; \quad$ // start time now double timeChange $=($ double $)($ now - lastTime $) ; / /$ find difference initial and final $(\mathrm{T})$

/*Compute all the working error variables*/ 
ISSN (online): 2581-3048

double error $=$ Setpoint $(\mathrm{SP})-$ Input $(\mathrm{PV}) ; \quad / /$ part

of proportional term

errSum $+=($ error $*$ timeChange $) ; \quad$ //integral term part

double $\mathrm{dErr}=($ error - lastErr $) /$ timeChange; //part

of

differential part

/*Compute PID Output*/

Output $(\mathrm{u})=\mathrm{kp} *$ error $+\mathrm{ki} *$ errSum $+\mathrm{kd} * \mathrm{dErr} ; \quad / / \mathrm{PID}$ equation in discrete time domain

/*Remember some variables for next time*/

lastErr = error; //previous error

lastTime = now; $/ /$ previous time

The value of constant $\mathrm{Kp}, \mathrm{Ki}$ and $\mathrm{Kd}$ was chosen to be 3.5, 0.45 , and 134 respectively that was depend on overshoots of the process plant and the oscillation period of the process plant when subjected to ON-OFF control. The PID of RTMFTRS was to maintained the temperature of the process plant within the temperature range of $38^{\circ} \mathrm{C}$ and $42^{\circ} \mathrm{C}$ with $40^{\circ} \mathrm{C}$ as the optimal or ideal performance. The behaviour of PID in system response of RTMFTRS is shown in Chart 1.

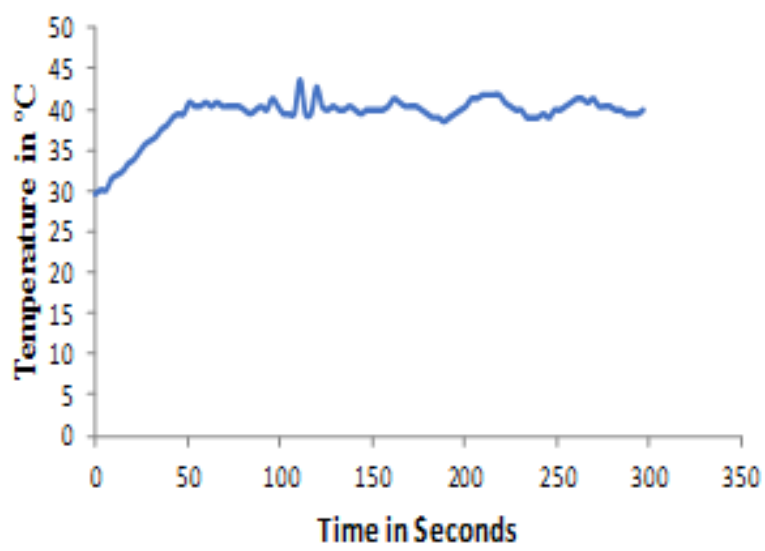

Chart 1: The PID response of the Plant

\section{V. $\mu$ CONTROLLER AND DISPLAY UNIT}

An Arduino Mega microcontroller was used in this study owing to its flexibility, availability and huge libraries database. It has fifty-four digital input/output pins and sixteen analog input pins each of which provide 10 bits of resolution (i.e. 1024 different values). Automation of the instrument was achieved with the aid of the microcontroller. The Liquid Crystal Display (LCD) is used to display the draught force during tillage operation for visual information. A Dig chip make 20 character $\times 4$ lines JHD162A liquid crystal display was used in the instrument developed. The display is a 16 pin which works with maximum power supply of $5.0 \mathrm{~V}$ and the data can be sent in either 4 bit, 2 operations or 8-bit, 1 operation so that it can be interfaced to 8-bits. Microcontroller used in 4 bits, 2 operation system [17, 18, 19].

\section{COMPLETE CIRCUIT OPERATION}

The Remote Temperature Monitoring Fault Tolerant Reporting System for Acid Gases Removal Unit (RTMFTRS) working function of Figure 4 as following:

i. The system monitoring temperature of the plant reporting any malfunction sending via modem SIM800 to delectated GSM number and alarm specific tone for temperature of abnormality for correction action according to plant configuration;

ii. The optodiac using first optocoupler, the anode of diode of the optocoupler 1 is connected to the MT1 of optodiac and gate of TRIAC as shown on Figure 5 is used to monitor optodaic failure. If optodiac is in normal operation, the output of optocoupler 1 will be will $\mathrm{HIGH}$ and the information will deliver to the delectated GSM reporting to state of optodiac if otherwise the output will be LOW reporting the failure and alarm with denoted tone for optodiac failure; and

iii. The second optocoupler was used to monitor the functionality and failure of the motor and TRIAC. Here, if Triac is open circuit the motor will not work and output of the second optocoupler will be LOW likewise, when motor is short circuit the anode and cathode of LED bridge the output will be LOW. Failure report will be sent to tell possible area where the fault is, via GSM module (SIM800) with corresponding alarm information. If in normal operation output be HIGH and appropriate information will be given telling state of the part of plant.

iv. If any fault in i. to iii. above is detected the main means cut-off through pin 35 of Arduino to the circuit breaker. The is sample fault information that will be send SIM800.

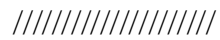

The void fault_analysis();

void send_sms_report();

char message[]= "";

char message1[]="It is either moc3021 is faulty or there is no input from ac source";

char message 2[]$=" I t$ is most likely there is no input from ac source";

char message3[]="Opto coupler 1 is bad";

char message4[]="Either Opto coupler 2 or the triac BT139 or motor is bad";

char message5[]="Either the temperature sensor or the heater is bad";

char message6[]="Either the temperature sensor is bad or there is no power to the sensor"; 
char message7[]="Either the temperature sensor is bad or there is no power to the sensor";

char message8[]="It is most likely there is no power to the sensor";

char message9[]="It is most likely sensor A0 is bad";

char message10[]="It is most likely sensor A1 is bad";

int send_sms $=0$;

int count $=0$;

int count $2=0$;

/////////////////////

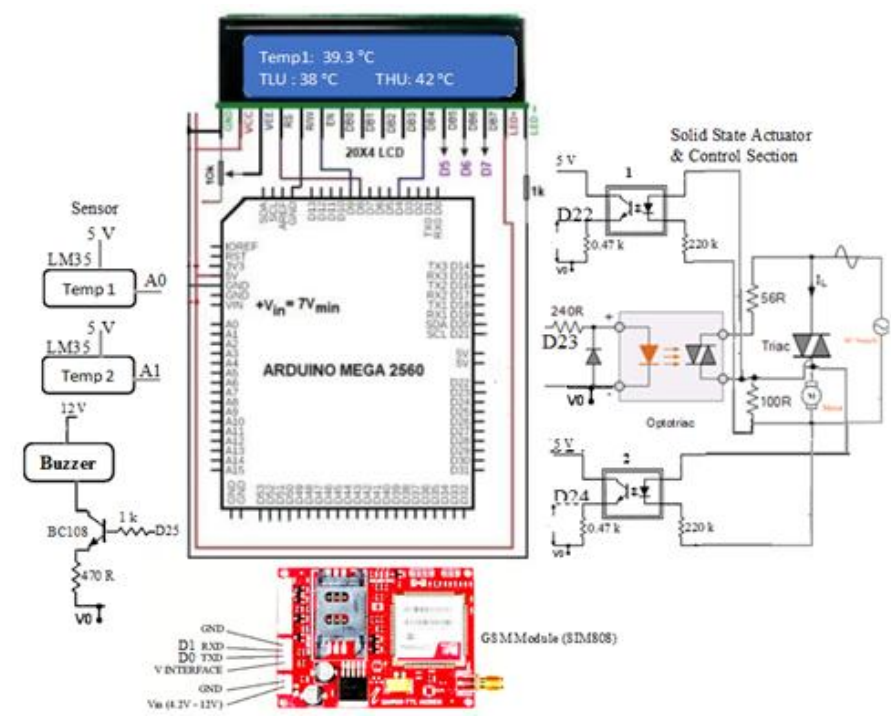

Figure 4: Complete Schematic Circuit of Remote Temperature Monitoring Fault Tolerant Reporting System for Acid Gases Removal Unit

\section{CONCLUSION}

The Remote Temperature Monitoring Fault Tolerant Reporting System (RTMFTRS) for Acid Gases Removal Unit is a fault diagnostic and tolerant scheme with a remote reporting feature to minimize system downtimes and allow the technician to carryout appropriate action to solved the faulty part. The fault diagnostic system is incorporated in a PID on RTMFTRS in order to provide control and fault management. The RTMFTRS was applied to a prototype Acid gas removal process requiring a temperature of $40^{\circ} \mathrm{C}$ for optimal performance with an acceptable range of $38^{\circ} \mathrm{C}$ and $42^{\circ} \mathrm{C}$. The remote monitoring was achieved using SMS to send fault reports to control system custodians as soon as the fault occurs. SMS was used to achieve a more reliable messaging system especially in areas of only $2 \mathrm{G}$ networks. Proetus was used to simulate the proposed developed system the result obtained work well and behaved as if is under normal condition.

\section{REFERENCES}

[1] Zhang, Y and Jiang J (2006). Issues on Integration of Fault Diagnosis and Reconfigurable Control in Active Fault-Tolerant Control Systems: International Federation of Automatic Control. 39(13) 1437-1448.

[2] Fekih, A and Pilla, P (2009). A New Fault Tolerant Control Strategy for Aircraft System Under Adverse Flying Conditions: International Journal of Control Automation and Systems. 3(2) 1-15.

[3] Bonivento, C., Paoli, A and Marconi, L (2001). Faulttolerant control for ship a propulsion system. European Control Conference (ECC). 1964-1969. doi: 10.23919/ECC.2001.7076210

[4] Escobet, T., Puig, V., Quevedo, J and Garcia, D (2014). A Methodology for Incipient Fault Detection: IEEE Conference on Control Applications (CCA), Juan Les Antibes, 2014; 104-109. doi: 10.1109/CCa.2014.6981336

[5] Wang, Y., Zhou, D., Qin, J.S and Wang, H (2008). Active Fault-Tolerant Control for a Class of NonLinear systems with Sensor Faults: International Journal of Control, Automation and Systems. 6(3), 339350 .

[6] Figueiredo, R, C., Ribeiro, A, M, O., Arthur, R and Conforti, E. (2011). Remote SMS Instrumentation Supervision and Control Using LabVIEW. 317-342 https://www.researcgate.net/publication/221914350 Accessed on 15/08/2020, 10:43 am.

[7] Omagbemi, O.W. and Nwkoye, A.O.C. (2018). Improving Proportional and Derivative Algorithms with Fault Diagnosis and Tolerance in Temperature Control Absorption System, Nigerian Journal of Physics, 27(1): 111-122.

[8] Rosa, P., Casau, P., Silvestre, C., Tabatabaeipour, S.M. and Stoustrip, J. (2012). A Set-valued Approach to FDI and FTC; Theory and Implementation Issues, 8th IFAC Symposium of Fault Detection, Supervision and Safety of Technical Processes, August 2012, Mexico. 12811286.

[9] Rodrigues, M, Theilliol, D. and Santer, D. (2005), Design of an Active Fault Tolerant Control and Polytopic Unknown Input Observer for systems Described by a Multi-model Representation, 44th IEEE Conference on Decision and Control, European Control Conference. ECC, Sevilla, Spain, Dec., 2005.

[10] Ewetumo, T. (2007): Development of Digital Display Dry and Wet Thermometer, International Journal of Physical Sciences, Nigeria. 2 (1):62-67.

[11] National Semiconductor (2016). Datasheet Catalog. Retrieved October 31, 2017, from Datasheetcatalog.com: 
ISSN (online): 2581-3048

Volume 5, Issue 10, pp 13-18, October-2021

https://doi.org/10.47001/IRJIET/2021.510003

ttp://www.datasheetcatalog.com/datasheets_pdf/L/M/3/ 5/LM35.shtml

[12] Popoola A. I, Akinpelu E. O, Ewetumo T (2021): Development and Performance Evaluation of an Intelligent Electric Power Switching System, International Journal of Trend in Scientific Research and Development (IJTSRD). 5(3): 65-69.

[13] http://www.openelectrical.org/wiki/index.php?title=Tri ac, Triac Basic Concepts. Accessed on 03- 09-2021, 21:36 pm.

[14] Jitha, P V and Unnikrishnan S K (2019). SMS Security System Using Encryption Techniques: International Journal of Computer Science and Mobile Computing (IJCSMC). 8(5) 132-142.

[15] Bret. (2011, April). Improving the Beginner's PID Introduction « Project Blog. Retrieved from http://brettbeauregard.com/blog/2011/04/improvingthe-beginner-pid-introduction/

[16] National Instruments. (2011). PID Theory Explained. Retrieved from http://www.ni.com/whitepaper/3782/en/ Accessed on 6/09/2021; 08:14 am.

[17] Arduino. (2015). Arduino uno. Retrieved September 19, 2017, from Arduino uno REV3: https://store.arduino.cc/arduino-uno-rev3

[18] Ewetumo T. and Orokhe J.E. (2019): Development of Paramagnetism Analyzer, Physical Science International Journal. India. 21(2): 1-12.

[19] Obagade T. A., and Ewetumo T. (2019): Development of Microcontroller Base Draught Force, Iconic Research and Engineering Journal (IRE). India 3(2): 476-480.

\section{Citation of this Article:}

Omagbemi O.W., Nwokoye A.O.C., "Development of Remote Temperature Monitoring and Fault Tolerant Reporting System for Acid Gases Removal Unit" Published in International Research Journal of Innovations in Engineering and Technology - IRJIET, Volume 5, Issue 10, pp 13-18, October 2021. Article DOI https://doi.org/10.47001/IRJIET/2021.510003 